\title{
A Dial-Reading Translator for Digital Machine Inputs
}

\author{
ARTHUR L. KLEIN \\ NONMEMBER AIEE
}

Synopsis: At the Southern California Co-operative Wind Tunnel, part of the need for rapid and accurate recording of instrument readings on tabular data sheets has been met by the development of a new automatic-translating device. This device has unusually low torque and is especially suitablefor use with self-balancing potentiometers. The device picks up the reading as a whole number and electrically transfers it to standard printing and punched-card machines, without lag and without restricting the normal operation of the potentiometer.

B ECAUSE of the high operating cost of a large transonic wind tunnel, such as the Southern California Cooperative Wind Tunnel, at Pasadena, Calif., it is of considerable importance that the experimental data be recorded as quickly and efficiently as possible without sacrificing reliability. The laborious and time-consuming task of permanently recording the many measurements required during the testing of models of modern airplanes hasled to the recent development of several automatic recording devices which pick off the reading or position of a force measuring instrument, translate the

Paper 52-118, recommended by the AIEE $\mathrm{Ai}$ Transportation and Computing Devices Committees and approved by the AIEE Technical Program Committee for presentation at the AIEE North Eastern District Meeting, Binghamton, N. Y., April 30-May 2, 1952. Manuscript submitted April 3, 1950; made available for printing February 28, 1952.

ARThUR L. KLEIN is with the California Institute of Technology, Pasadena, Calif. Kennetr P. Gow was with the California Institute of Technology, Pasadena, Calif., at the time the paper was written, and is now with North American Aviation, Inc., Downey, Calif.

The authors are indebted to members of the staff of the Co-operative Wind Tunnel and of the Guggenheim Aeronautical Laboratory at the California Institute of Technolc a help and suggestions. J. E. Smi Director of the Co-operative Wind Tunnel, made especially important contributions to the present paper.

\section{KENNETH P. GOW \\ MEMBER AIEE}

reading into a whole number, and then print the number on a data sheet. However, to the authors' knowledge, none of these devices could be adapted easily or efficiently to reading electric straingauges.

Increasing use is being made of small balances, mounted inside the aircraft model, which have strain gauges as the force sensitive element and for which a self-balancing potentiometer is the forceindicating instrument; see Figure 1. For normal tests at the Co-operative Wind Tunnel a battery of three to eight of these potentiometers, representing three to eight forces or moments and requiring the continuous attention of two to five technicians, must be read for each model configuration, model attitude, and Mach number. Clearly it would be advantageous and profitable to employ reliable automatic translators of potentiometer readings which would require the attention of only one technician regardless of the number of channels being read. Such a device has been developed at the Cooperative Wind Tunnel and is described in this paper. It eliminates manual recording from potentiometers of the selfbalancing type. The strip-chart type of instrument is not satisfactory because it is less accurate than the dial-indicating type and because its record must be read and manually re-entered on data sheets or punched cards for further calculation.

\section{Design Principles}

In June 1947, the senior author undertook the design of a translator suitable to the needs of the Co-operative Wind Tunnel operations. The design principles which he set up were essentially as follows:

1. The translator should pick up the read- ing of a standard, precision, self-balancing potentiometer and transmit that reading as a whole number, either positive or negative, to a standard punched-card tabulating machine.

2. The translator should follow the movement of the potentiometer without lag even though a printing cycle is taking place.

3. Standard parts should be used as much as possible, wear and maintenance must be minimized, and the dragging torque imposed on the potentiometer must be negligible.

4. The translated reading must be clear and unambiguous.

By December 1947, the basic design work was completed by the senior author and during the following year the details, including the double brush system for tens transfer and the double zero elimination, were for the most part filled in by the junior author. In May 1949, the operation of the basic components was checked out with a temporary gear system, and by November 1949 the completely assembled system was operating satisfactorily.

\section{General Arrangement}

The over-all mechanical and electric system for a typical model test is shown

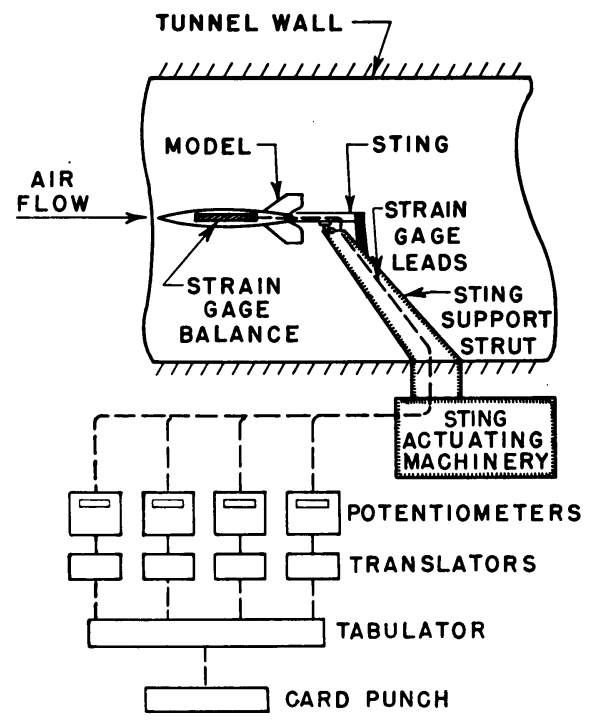

Figure 1. Typical wind tunnel model internal balance installation 
POTENTIOMETER

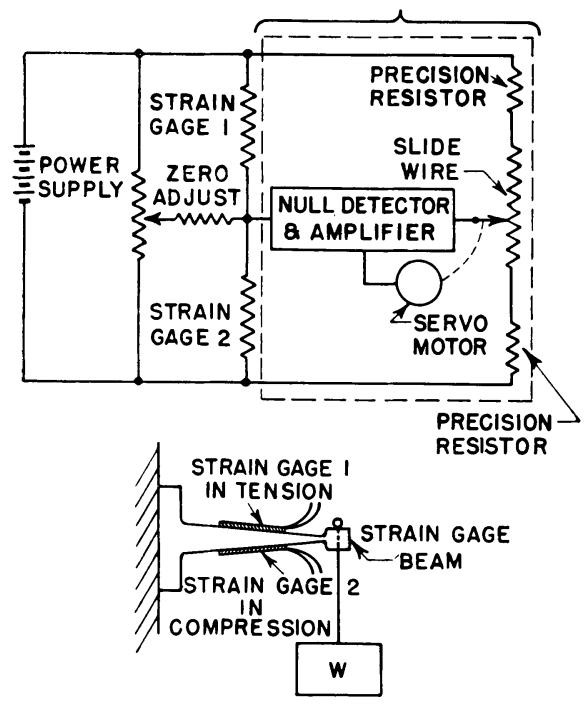

Figure 2. Typical strain gauge and potentiometer circuits

schematically in Figure 1. Figure 2 shows the circuit diagram for a typical strain gauge setup. These systems are quite common and will not be discussed here. Actually the translator interprets only the potentiometer dial reading and does not care what electric signal is fed into the potentiometer. The connection between the two is purely mechanical, and, in fact, the translator is not restricted to use with a self-balancing potentiometer, but may be used to indicate the angular position of any rotatable shaft provided sufficient driving power is supplied by the shaft. It is preferred, but not necessary, that the potentiometer dial be a linear scale. If the scale is nonlinear, however, it is possible to make the proper adjustment in the data reduction process.

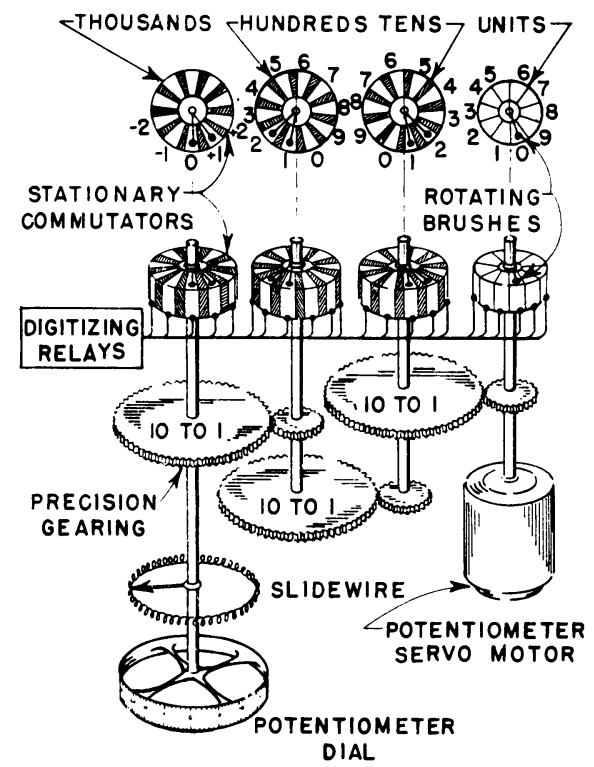

Figure 3 is a simplified sketch of the physical arrangement of the translator. A series of spur gears is connected directly to the shaft of the potentiometer servo motor which also turns the potentiometer slidewire and dial to the balance position. Precision-built gears are utilized to reduce the friction torque and minimize maintenance. By the proper selection of bearings and gears and by careful alignment of the gear train, it was found that a backlash removal system was unnecessary. The entire gear train is mounted inside an aluminum alloy casting which is bolted to the chassis of the potentiometer. To the outside of this gear box are mounted four nonrotating commutators, serving as switch contacts, each concentric with a gear shaft as shown in Figure 3. The center of each commutator has been bored out to permit the extension through it of the gear shaft. To these protruding ends of the gear shafts are attached the brush holders and their silver graphite brushes, the brushes riding on the ends of the commutators. Electric power is supplied to one rotating brush on each gear shaft through the end of the shaft which makes direct contact with a flat band of phosphor bronze; this type of rotating contact requires very little driving torque because it operates at such a small radius. For the three commutators which have double brushes, see Figures 3 and 4 , power is supplied to the second brush through a conventional slip ring arrangement mounted near the end of the shaft. By using the proper combinations of gear ratios and number of commutator segments it is readily apparent that the position of the rotating brushes can be made to be equivalent to the position of the potentiometer dial.

If we assume, for example, that the entire length of the potentiometer dial is divided into 10,000 equal divisions and that the over-all gear ratio between the dial or slidewire shaft and the units counter gear shaft is 1,000 to 1 , then one revolution of the units gear shaft corresponds to a dial travel of ten divisions, and, similarly, one-tenth of a revolution of the units gear shaft is equivalent to a dial travel of one division. Now, if the units commutator has ten equal segments, the movement of the rotating brush in going from one segment to the next represents a movement of one division of the potentiometer dial or a "count of one" change in the dial reading. If each segment is wired to a lamp in such a way that the rotating brush or switch completes the electric circuit through a segment and its lamp when making contact with that segment, then as the dial traverses the units brush rotates and causes each segment light to flash in succession with the cycle being completed once every ten dial counts. In similar manner light circuits can be set up for the tens, hundreds, and thousands commutator counters so that one light in each counter will be lit at a given dial position and the group of four lights will indicate the dial reading to the nearest division.

The physical arrangement just described is quite conventional, being used in automobile mileage indicators in the tenths column, watt-hour meters, and so forth. But such a simple electric circuit could not be used because the reading is not definite; that is, two lamps in the same counter could be energized simultaneously, the units and tens counters would not necessarily reach a count of ten simultaneously, the units, tens, and hundreds counters would not necessarily reach a count of 100 simultaneously, and so forth. In other words, when the potentiometer dial reading is 099 this simple lamp circuit might show such readings as $009,090,109,199$, and so forth. To ensure that the correct reading

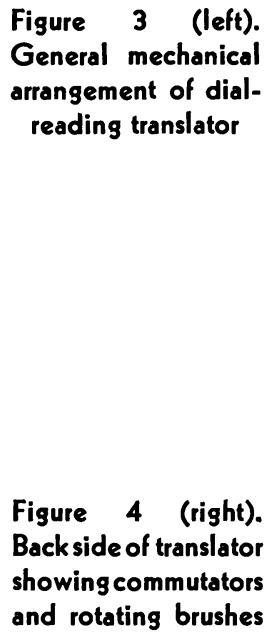

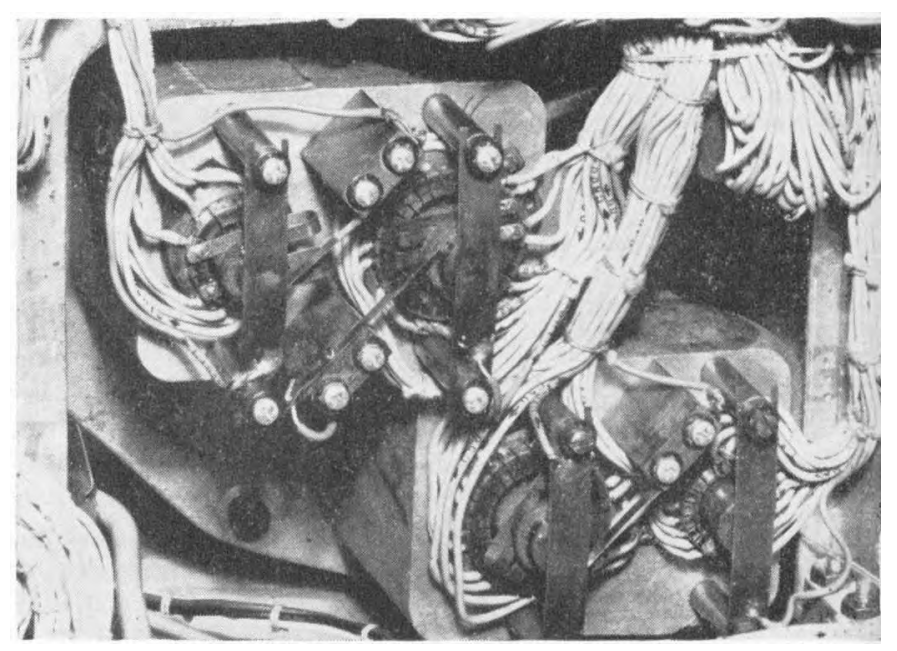



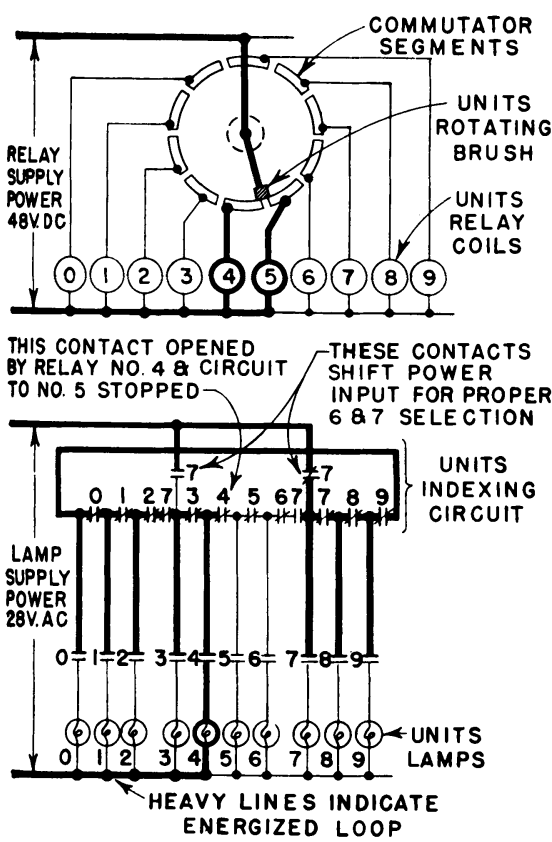

Figure 5. Circuit for digitizing units, shown at a count of 4

- represents an open contact unless the relay coil is energized

$\neq$ represents a closed contact unless the relay coil is energized

is given by the lamps, it is necessary to superimpose control circuits upon the lamp circuits which will prevent erroneous lamp readings. The control circuit (considering now the process of the dial reading going from 099 to 100) must hold the hundreds lamp reading at 000 and the tens lamp reading at 90 until the units reading of 9 changes to 0 ; then simultaneously with the change from 9 to 0 in the units lamps the tens lamp reading must change to 00 and the hundreds lamp reading must change to 100 . These control circuits are called indexing systems and are described in the next section.
One other rotating part which is included in the translator is the a-c drag cup induction generator. This generator acts as a damper on the motion of the servo motor and is directly connected to the motor shaft. The generator output, which is manually controllable through a voltage divider, is connected to the potentiometer amplifier and modulates the signal of the amplifier to the servo motor, thereby giving an effective dragging action proportional to the motor speed.

The inertia of the entire translatorpotentiometer system is not more than 10 per cent greater than that of the potentiometer in its original condition, and the frictional torque is about the same; note that originally the potentiometer had a worm gear drive. Thus the speed of response and the sensitivity of the potentiometer have not been modified appreciably.

\section{Indexing Methods}

With the mechanical arrangement of Figure 3 it is clear that when a rotating brush makes contact with one and only one commutator segment a closed electric circuit can be formed, including an indicator light, which will indicate the number of the commutator segment on which the brush is resting. But when the brush is midway between two adjacent segments the brush will contact both segments, or neither, depending upon whether the width of the brush face is larger or smaller than the gap between the segments. If the brush is wider than the gap both lamps will be lit, while if the brush is smaller than the gap no lamps will be lit. Certainly neither of these conditions is tolerable in our case even though the true correlation of brush position to potentiometer dial reading requires that the

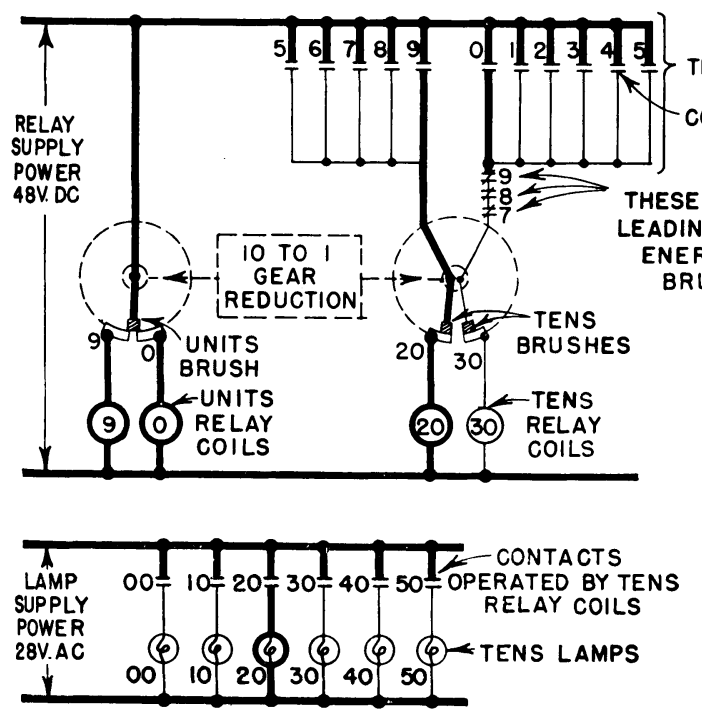

TENS INDEXING CIRCUIT

CONTACTS OPERATED BY UNITS RELAY COILS

THESE UNITS CONTACTS PREVENT THESE UNTS CONTACTS BROM BEIN IS DE-ENERGIZED.

Figure 6 (left). Circuit for controlling the tens transfer, shown at a count of 29

Figure 8 (right). Units indexing, complement number, and lamp circuits at a reading of $(-8)$
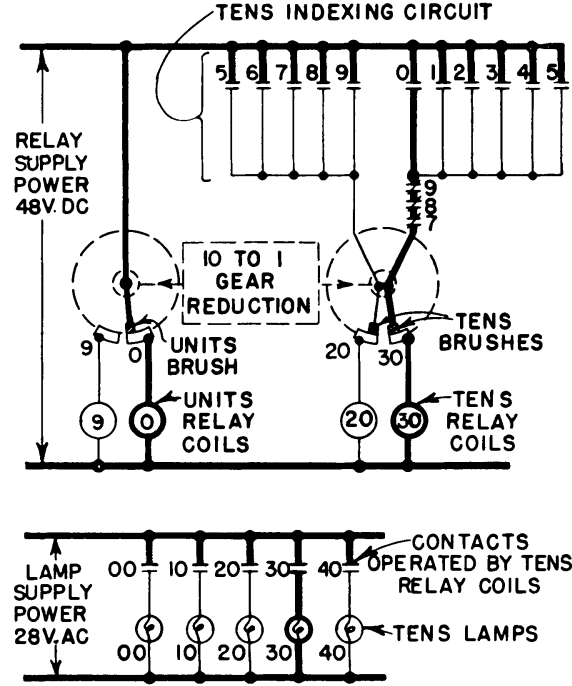

Figure 7. Circuit for controlling the tens transfer, shown at a count of 30

brush be midway between the two segments. A choice of numbers must be made either manually or automatically. A manual method would defeat the purpose of the instrument. Therefore, an automatic method must be devised to make the instrument pick one number or the other and do it consistently. This requires a "snap action" or indexing system. The method of indexing probably is the most important feature of any translator.

Mechanical indexing devices, for example, the Geneva motion system used in automobile mileage counters, are subject to rapid wear because of sliding surfaces and impact loads and have rather low speed limits because of the high accelerations encountered. For instance, one can

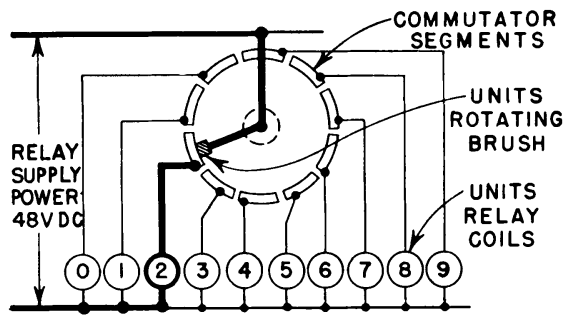

THIS CONTACT OPENED THESE CONTACTS BY RELAY NO 2 Q CIRCUIT SHIFT POWER

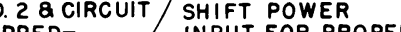
TO NO. 3 STOPPED
687 SELECTION

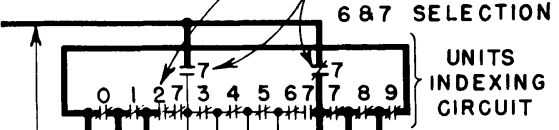

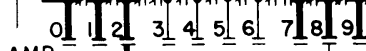

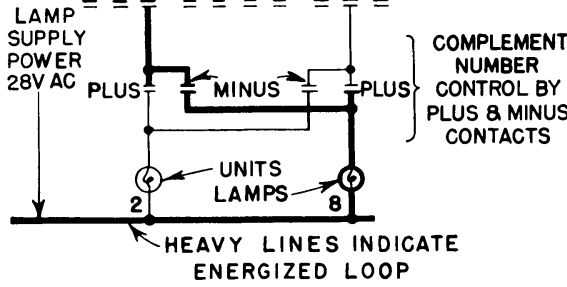




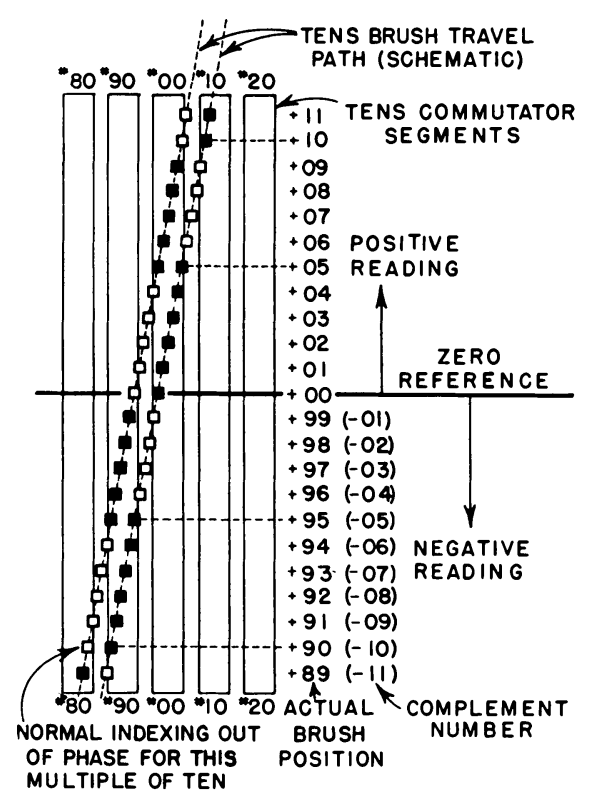

- DE-ENERGIZED TENS BRUSH - ENERGIZED TENS BRUSH

Figure 9. Schematic layout of tens brush positions for positive and negative readings

visualize easily the difficulties which would be met if the rotating brush were required to jump rapidly from segment to segment so as to prevent contact with two segments at the same time. For these reasons it was decided to delegate the all important indexing action to electric relays, a decision which was supported further by the knowledge that multicontact relays were required to provide additional circuits for interlocking and monitoring functions. The particular circuit chosen is shown in its simplest form in Figure 5.

The upper half of Figure 5 is a schematic end view of the units rotating brush and commutator arrangement with the brush making contact with both number 4 and 5 commutator segments. Note that in the figure the brush is shown inside the commutator for purposes of clarity. Power is supplied to both segments by the brush and thereby to the number 4 and 5 units relay coils. These relay coils operate correspondingly numbered contacts in a circuit (see the lower half of Figure 5) which is energized along the path shown by the heavy lines. The lower portion of Figure 5 shows the units indexing circuit connected to a bank of lamps and allowing only the number 4 lamp to be lit. All contacts in this indexing circuit are shown in their normally de-energized position. With the rotating brush making contact with both number 4 and 5 commutator segments, all number 4 and 5 contacts in the indexing circuit are energized and therefore their normally open or closed positions are reversed. Study of the circuit will show that current can pass through only the number 4 lamp; the circuit to the number 5 lamp is blocked by the combination of the number 4 and 7 contacts. As soon as the rotating brush moves completely off of the number 4 commutator segment going toward the number 5 segment, the number 4 units relay coil is de-energized and all number 4 contacts revert to their deenergized positions, thereby leaving a clear circuit through the number 5 lamp. Similarly, each successive lamp will be lit as the brush revolves through a full revolution with the lower number always taking preference. The indexing loop or circle is always broken by the four special number 7 contacts, with the left-hand power input line being used only when the brush
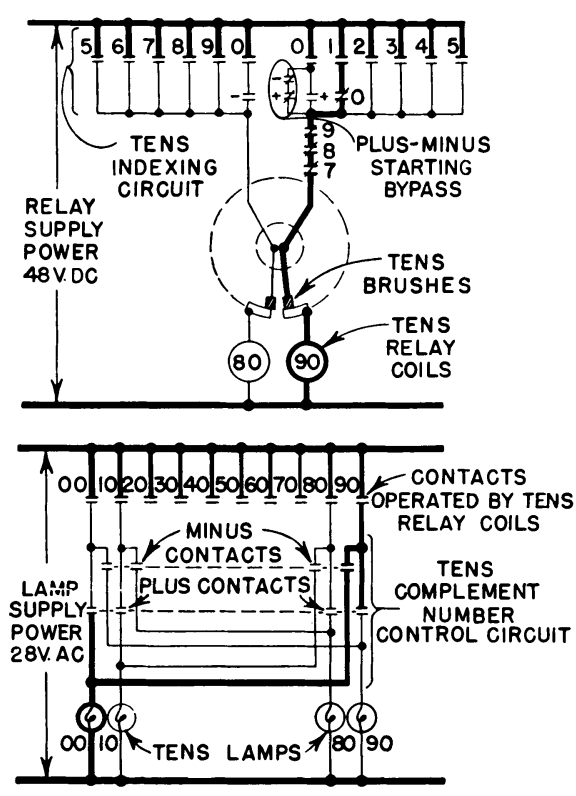

Figure 10. Circuit for controlling the tens tuansfer, shown at a reading of $(-9)$

touches the number 7 commutator segment. The time required to transfer from one units count to the next is either a relay pull-in or drop-out time, not both. A parallel units indexing circuit, not shown, is provided for supplying number signals to punched-card machines.

From the units indexing system we now progress to the system used for the tens brush-commutator counter. If the same system were used as for the units counter it is readily apparent that the length of the tens-brush rotating arm would have to be roughly ten times the length of the units-brush arm to maintain the same sensitivity. Since this would impose low maximum speed limits and would require much more space, a somewhat different approach is used in which two rotating brushes are mounted on the tens gear shaft (see Figures 3, 4, 6, and 7). The width of each tens brush is less than the length of the gap between commutator segments, the spacing between the brushes is less than the width of one segment, and the length of the gap is less than the segment width. Thus, one of the brushes always will be in contact with a segment; both brushes may contact the same segment, the two brushes may contact different segments, or one brush may contact a segment while the other brush is in between segments. Then by switching, or indexing, the power supply from one brush to the other at the proper time, it is always possible to energize the correct tens relay coil and indicator lamp. This indexing is controlled by the units counter, by the circuit shown in Figures 6 and 7 , thereby ensuring that the sensitivity of the tens count is equivalent to that of the units count. The double brush system requires two more brushes (one brush rotating on the commutator and one slip-ring brush for power delivery) than does the single brush system but the rubbing speeds are only onetenth as large.

The diagrams of Figures 6 and 7 show a simplified layout of the tens brush and indexing system with the circuits set up for counts of 29 and 30 respectively. A portion of the units commutator and relay system is included for reference. Again it should be noted that the brushes are shown inside the commu ator for reasons of clarity. Contrary to the units system, the tens indexing circuit controls

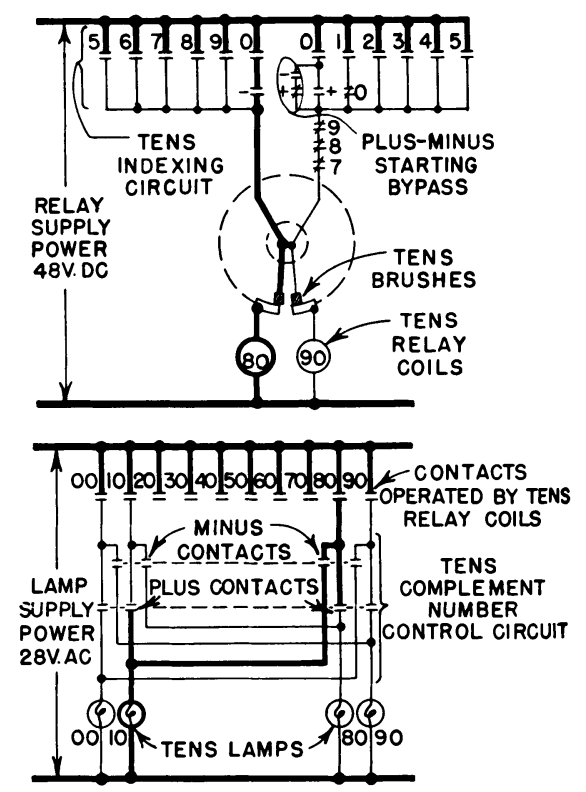

Figure 11. Circuit for controlling the tens transfer, shown at a reading of $(-10)$ 
the relay power transmission ahead of the rotating brushes and is simpler because it is only necessary to index, that is, switch the power supply from one brush to the other when the units count goes from 9 to 0 or vice versa.

In Figure 6 the units rotating brush is shown contacting units positions number 9 and 0 with the result that units indicator lamp number 9 is energized in the same manner that lamp number 4 was energized in Figure 5. At the position shown in Figure 6, both the number 9 and 0 normally open contacts in the tens indexing circuit are closed, and a number 9 normally closed contact, now open because units relay coil number 9 is energized, is blocking the circuit to the leading (right hand in Figure 6) tens brush so that only the number 20 tens relays coil is energized and the number 20 tens lamp is lit. Transfer, or indexing, in the tens decade occurs, as shown in Figure 7 , when the units brush has just broken contact with units position number 9 and is on position number 0 . At this position, the units number 9 relay coil is de-energized which opens the only circuit to the trailing tens brush and completes the circuit to the leading tens brush, thus energizing the number 30 commutator segment and relay coil and lighting the number 30 lamp. As indicated in Figures 6 and 7, units counts of $5,6,7,8$, and 9 energize the trailing tens brush, and units counts of $0,1,2,3,4$, and 5 energize the leading tens brush. At the number units position, both tens brushes contact the same tens commutator segment and the tens indexing circuit allows both brushes to be

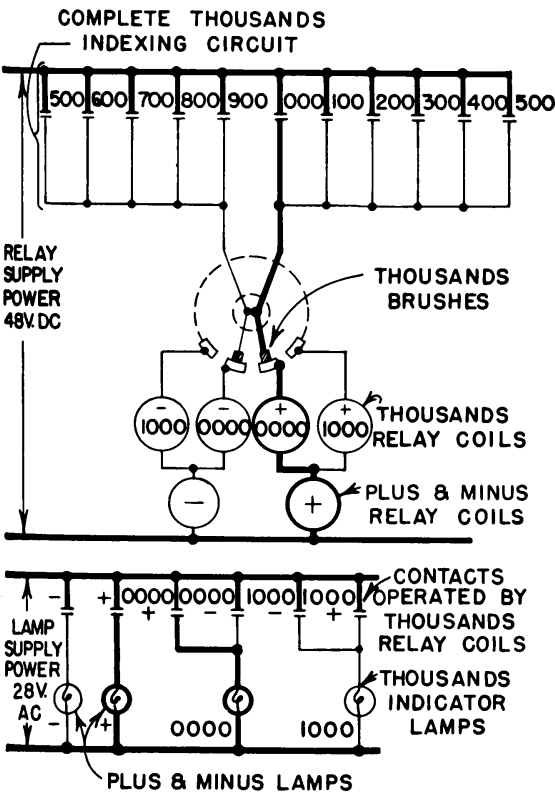

Figure 12. Circuit for controlling the thousands and plus-minus transfer

Figure 13. Prototype dialreading translator set up for operational tests in a 2-channel mockup cabinet

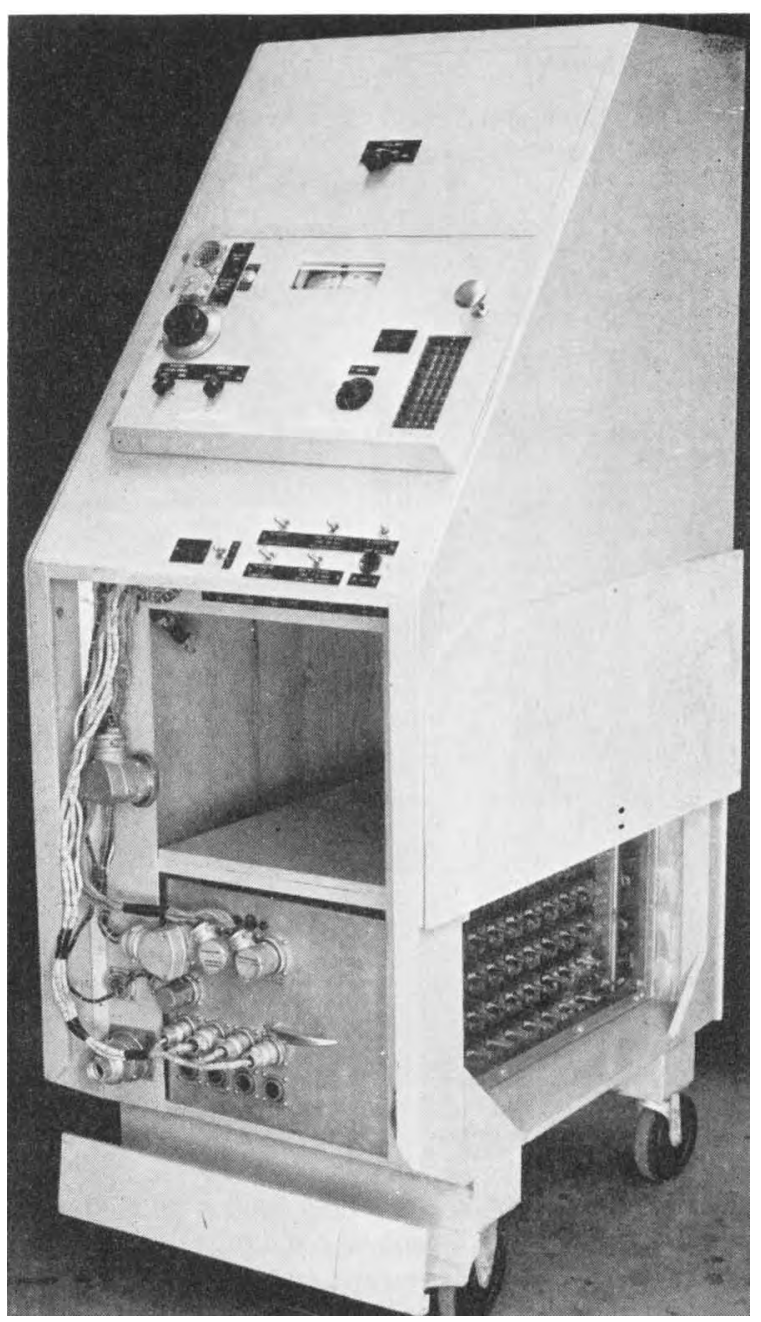

energized, thereby increasing the tolerance on brush position in the tens circuit.

The indexing systems for the hundreds and thousands counters are similar to that used for the tens counter, in that the same type of double brush circuit is employed, with the indexing circuit controlling the energizing of the brushes. The tens relay coils operate the contacts in the hundreds indexing circuit, and the hundreds relay coils operate the contacts in the thousands indexing circuit. Thus, in effect, the units relay coils control the indexing throughout the entire system and by that means establish that the sensitivity of the entire system will be equivalent to the sensitivity of the units counter.

\section{Discussion of Basic Design}

In the preceding paragraphs the basic design of the translator has been described in considerable detail. The major advantages of this design can be stated as follows:

1. Speeds greater than the maximum continuous counting speed (relay response time) do not cause damage. The maximum rate of brush travel, in the units counter, is nearly 600 counts per second, which figure is determined by the desired number of counts across the potentiometer scale and the maximum rpm, 1,800 , of the potentiometer servo motor. The relays follow at speeds up to about 50 counts per second.

2. The correct count or reading is obtained automatically after a high speed, greater than 50 counts per second, run when the balance position is reached, irrespective of the number of counts missed by the relays. This means that the translator does not restrict the motion of the potentiometer and that it does not have to count every number when traversing from one position to another.

3. The dragging torque of the translator is independent of the potentiometer dial position.

4. The system counts down as easily as up

5. Indexing, which requires snap action by some mechanical part, is handled by standard relays and contactors which have had many years of development to achieve reliability in this type of operation.

6. If the translator power is interrupted for any length of time the correct count is immediately re-established when the power is restored.

7. It is believed that the maintenance requirements of the translator will be small.

8. The translator may be adapted easily to many other data producing systems. 


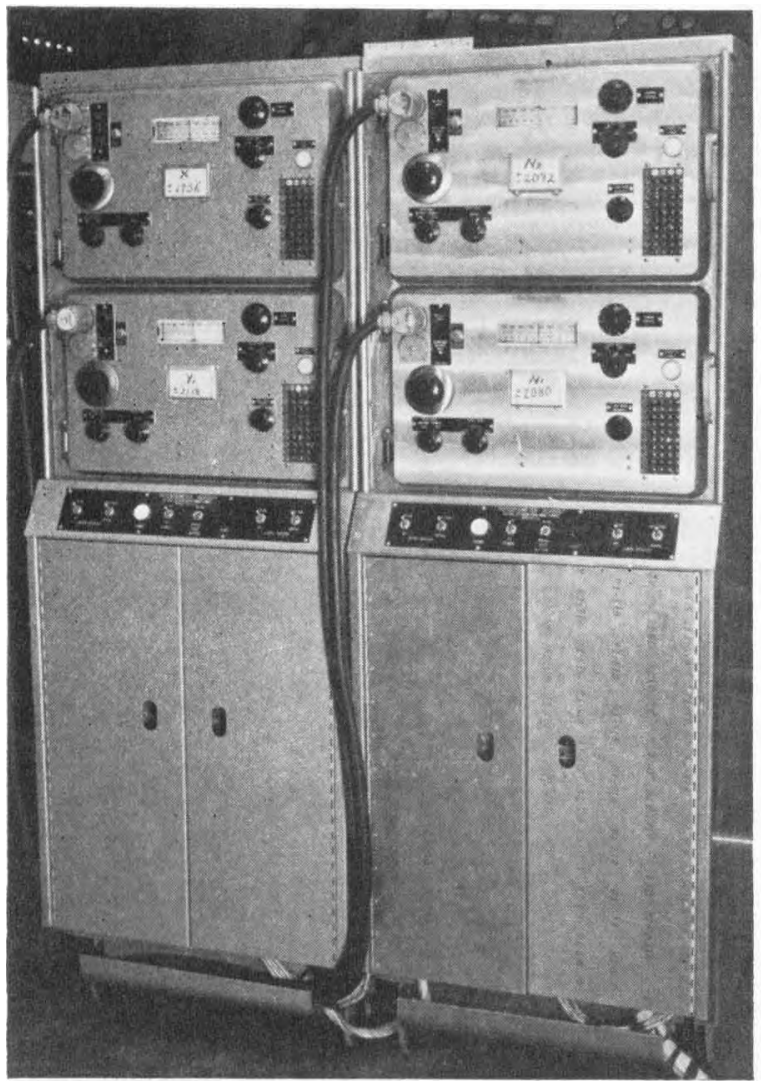

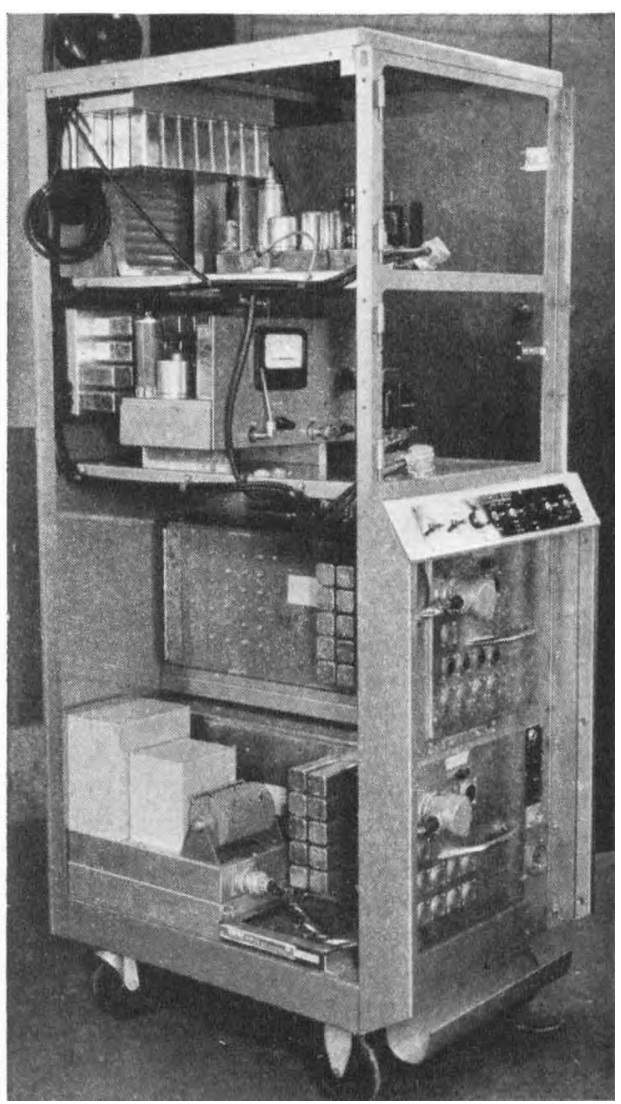

In constructing the prototype unit it was found more convenient to use a gear reduction of 1 to 5 between the units and tens shafts than the 1 to 10 ratio shown in the sketches, and the number of units counts or segments on the units commutator was increased to 20 , that is, the units brush goes from 0 to 9 twice in one revolution.

The thousands counter is used primarily to control the transfer between positive and negative readings (see discussion in next section) rather than to give readings to four figures. The accuracy of the potentiometer itself is not equal to four significant figures, but, for the sake of clarity in reading, the full dial range is divided into approximately 2,100 counts.

The diagrams in this paper show only the circuits for the count-indicator lamps. In the prototype device a parallel circuit is provided in every case to furnish the count signal to International Business Machines card-punching and printing machines. Still other parallel circuits could be added if desired.

\section{Method for Indicating Negative Numbers}

In a normal wind tunnel test the strain gauge will indicate both positive and negative forces, so that it is desirable to read and record the data with the proper reference zero. Circuits have been in- cluded in the translator which enable it to indicate negative as easily as positive numbers. These circuits are described in the following paragraphs. It should be noted that Figures 5, 6, and 7 show only those circuits required for positive number indication.

First, assume that the potentiometer is zeroed at the center of its dial and that the translator counters are set to read zero simultaneously. Then if the strain gauge input is increased in the positive direction the translator lamp bank will show in succession the numbers: 0000 , 0001, 0002, 0003, and so forth. But if the strain gauge output increases negatively, the potentiometer dial moves in the opposite direction and the translator lamp bank (assuming the negative number circuits have been omitted) will show in succession the numbers: 0000, 9999, 9998, 9997, and so forth. Thus it is necessary to add additional controls which will convert these latter numbers to their complements and also will give a minus sign indication. It is clear that if every individual lamp circuit is connected with its complement (for example, number 9 with 1,8 with 2,60 with 40,400 with 600 , and so forth) the addition of plus and minus contacts in these branch leads will give control of the choice between a number and its complement. The arrangement for the units count is straightforward and is shown in Figure 8. A pair of p'us and minus normally open contacts is inserted in each of the number 1 , $2,3,4,6,7,8$, and 9 lamp circuits (note that the complements of 0 and 5 are 0 and 5 ). Only the circuits for the number 2 and 8 lamps are given in Figure 8. When the reading is positive all plus contacts are closed, and when negative, all minus contacts are closed. Thus if the units brush is at the number 2 commutator segment but the reading is actually negative so that the minus contact is closed (plus contact open), the circuit will be closed through the number 8 lamp and a count of $(-8)$ will be indicated on the lamp bank.

In the tens-counter circuits the arrangement is somewhat more complicated because the indexing is accomplished ahead of the double rotating brushes rather than after the brush as in the units system (for example, compare Figures 5 and 6), and because the indexing system as shown in Figure 6 and 7 will be out of phase at every multiple of ten when the reading is negative. This phase shift is demonstrated in Figure 9.

Figure 9 shows in schematic form the physical position of the tens brushes for the first 11 counts in both the positive and negative directions. The left-hand column of numbers shows the lamp bank readings which would be obtained with the circuits of Figures 6 and 7 . The righthand column of numbers, negative half 
only, shows the correct readings which are desired. Inspection of this array will show that paralleling the number 90 and 00 tens-lamp circuits and inserting plus and minus contacts will give the correct complement number reading (assuming, of course, that the units system of Figure 8 is in operation) for the first nine counts but not for the tenth count. Similarly, paralleling the number 80 and 10 tenslamp circuits gives the correct complement for the counts of $89(-11)$ to 81 (-19) inclusive, but not for the 20th count. Thus we see that at every tenth negative count the device of paralleling the lamp circuits is inadequate in that the entire system is out of phase by one count. The proper phase relationship could be obtained if an extra count $(-00)$ were added between +00 and -01 , but this is undesirable because it puts an error in the numbering system and coarsens the zero setting. The method finally adopted was to set up two (0) circuits in the tens indexing circuit with one controlled by a plus contact and the other controlled by a minus contact. The plus (0) circuit is the same as that shown in Figures 6 and 7 and is used only for positive numbers and the reference zero. The minus ( 0 ) circuit is used only for negative numbers and has the effect of causing the tens system to index between the 1 and 0 units counts instead of between the 0 and 9 units counts, which effect is the same as the desired phase shift of one count. In Figure 9 this shift would result in the lefthand brush being energized at the number 90 brush position instead of the right-hand brush and, therefore, the number $80 \mathrm{com}$ mutator segment being energized instead of the number 90 segment, thus enabling the correct reading of -10 to show on the lamp bank. The tens indexing circuit with the positive-negative controls added is shown in the upper half of Figures 10 and 11 for readings of -09 and -10 .

In the lower half of Figures 10 and 11 are shown the paralleled tens-lamp circuits with the plus and minus control contacts. Again only part of the lamp circuits is shown to avoid confusion. Note that the normally closed ( 0 ) contact in the (1) circuit of the tens indexing system is required (for negative readings) to prevent the leading tens brush from being energized through this (1) circuit when the units brush is contacting both the number 0 and 1 units commutator segments.

If a diagram similar to Figure 9 is drawn up for the hundreds and thousands counters it will show that the count does not get out of phase as did the unmodified tens counter. This means that the cor- rective plus-minus circuits of the tens indexing system should not be added to the indexing systems of the hundreds and thousands counters. In fact, the latter must be analogous to the simple tens indexing circuits shown in Figures 6 and 7. However, the complement number circuits in the lamp bank system are similar in all three double-brush counters (see Figures 10 and 11).

To complete this discussion of the control of positive and negative numbers, we have only to mention the method used to actuate the plus and minus contacts; that is, the means used to determine the algebraic sign of the potentiometer reading. Of all four counters only the thousands counter does not rotate more than 180 degrees in either direction; and therefore only in the thousands counter will a commutator segment always be positive or negative. In Figure 12, the circuit arrangement for the thousands counter is shown. When the reading is positive one of the two right-hand commutator segments is energized and a plus signal is given; and when the reading is negative one of the two left-hand segments is energized and a minus signal is given. These plus and minus relay coils control all plus and minus contacts in the translator. If all counters are at zero the leading (right-hand) thousands brush is energized and a plus signal is given; then if the units counter moves negative by one count to the $(-1)$ position the trailing (left-hand) thousands brush is energized, the minus relay coil is energized, and units, tens, and hundreds circuits switch to their complement number systems.

One other characteristic of these systems is that if the relay power supply is turned on with the units or tens counters at their zero position the plus-minus contacts will block the signal to the higher counters and the plus-minus relay coils will not be energized. For example, if the power is turned on with the tens counter at (00) the thousands brush and plusminus coils will not be energized because the plus-minus contacts in the tens indexing circuit block the circuit to the thousands brush. To remove this difficulty a plus-minus, starting by-pass circuit (see Figures 10 and 11) is included in the tens indexing circuit, thereby allowing the correct plus or minus relay coil and contacts to act, after which the by-pass takes itself out of action.

\section{Automatic Averaging of Fluctuating Balance Loads}

One important characteristic of strain gauge signals is the nearly complete ab- sence of any damping in the gauge. If, as is usually the case, the strain-gauge, model-force balance also has no internal damping, then the strain gauge signal coming to the potentiometer frequently will oscillate rapidly about the mean value. Two special devices are included in the translator-potentiometer combination which enable the wind tunnel operator to obtain an average reading.

One device is the 60-cycle a-c drag cup induction generator which is mounted in the translator gear box and whose armature is driven by the potentiometer servo motor with a 1 to 1 speed ratio. The generator output, which is directly proportional to the revolutions per minute of the servo motor, is put into the potentiometer amplifier in such a way that the strength of the amplifier signal to the servo motor is reduced. By this means the response of the motor is reduced and its ability to follow the strain gauge oscillations is curtailed. Enough damping action is provided so that with full damping the potentiometer metrical circuit unbalance required to produce full servo motor speed is several times the unbalance required with damping.

The other device is a low-pass filter in the strain gauge signal input. All force oscillations with frequencies above $1 / 2$ cycle per second can be attenuated and in most tests effectively eliminated. Several filters are provided, each with different cutoff frequencies, and various combinations of them may be selected as desired. The filter circuit does not modify the speed of response of the servo motor.

These two devices have been in operation in routine wind tunnel tests and have proved to be quite satisfactory as well as necessary.

\section{Additional Features}

Several other features of the translator are described briefly now.

The indicator lamps are combined in a 4-column bank of the instrument panel (see Figure 13) and light up numbered jewels. Provisions for operating a remote lamp bank are provided.

Data printing circuits are paralleled with the indicator lamp circuits and connected to International Business Machines standard punch-card machines. The printing cycle is controlled by a push button. Plus and minus signs are shown on the lamp banks and recorded in the printing cycle with the numerical reading. When the printing cycle is initiated all commutator relays are locked up to hold the reading while the brushes continue to rotate and follow the potentiometer read- 
ing, that is, the printing operation does not interfere in any way with the normal action of the potentiometer. The lamp bank reading is also "locked up" during the printing cycle. Additional circuits are provided which prevent printing a reading in the event that there is no number or more than one number in any of the four decades; this condition may exist momentarily during high-speed travel of the potentiometer dial and translator, or because of faults in the relays.

The potentiometer scale zero reference may be placed at the center of the dial or displaced to fixed positions at either end of the dial. This amounts to adding a "pan weight" to the reading. The translator thousands counter has a special circuit which causes the translator to indicate the correct reading regardless of which zero reference is used.

\section{Conclusion}

Figures 14 and 15 show two views of the 4-channel chassis.
The prototype translator, shown in Figure 13, has been used to record data in many routine wind tunnel tests and has operated quite satisfactorily. The translator cabinet shown in Figure 13 is a wooden mockup and is to be replaced by a steel frame. The Co-operative Wind Tunnel is constructing enough additional units to enable it to record automatically all strain gauge balance data. It is believed that this device will prove to be a very useful addition to wind tunnel instrumentation.

\section{Discussion}

Harry E. Burke (Consolidated Engineering Corporation, Pasadena, Calif.): I find this paper very interesting, particularly because I have had the opportunity to examine the equipment as it was operated in conjunction with the Co-operative Wind Tunnel. As far as I have been able to ascertain, the unit described is entirely satisfactory in this application. The method of damping out cyclical variations imposed on the input signal is straight-forward and is a very useful feature. The required toggle action in stepping from one digit to the next is accomplished in a relay matrix which also is used to store a particular digital reading while the next reading is being set up on an analogue basis. This also has some very important advantages in some applications.

The only criticisms that I can make are relatively minor ones and are not intended as reasons for curtailing the use of this equipment. The use of an analogue section and a digital section in this one instrument appears to be rather expensive and it is felt that if these functions could be combined together in some way then the cost of digitizing the input signal would be reduced greatly.

It is my belief that the equipment as it is described would be capable of an accuracy and a linearity of about \pm 1 part out of a possible full scale' of 1,000 parts. As this accuracy depends in a great part on the slide-wire used in the analogue section and in the gear train driving the digital section, it seems to me that there would be a deterioration in these specifications as the slidewire and the gear train wear with use.

I found the demonstration of this unit as a standard part of the Co-operative Wind Tunnel's instrumentation very interesting and I would like to congratulate the authors of this paper for its excellent design.

Arthur L. Klein and Kenneth P. Gow : Mr Burke's statement in his second paragraph is quite correct. The instrument would be much simpler without the potentiometer and its accessories. These particular instruments were designed to be used with strain-gauge force measuring equipment. A simple digitizing unit would require only the gear box and, without special features, only four relays. The inaccuracies in the instrument lie almost entirely in the potentiometer. The gear train commutators and their equipment have intrinsic accuracies of at least two orders of magnitude higher. The writers believe that with proper precaution a gear box system, as described, can be made with an error of less than 0.0001 per cent. 